\title{
Adaptive Group-based QoS Aware Link State Proactive Multipath Routing for Large-Scale MANETs (GMP-OLSR)
}

\author{
Kebebew Ababu Yitayih* \\ Faculty of Computing, Jimma University, Ethiopia
}

\section{Article Type: Article \\ Article Citation: Kebebew Ababu Yitayih. Adaptive group-based QoS aware link state proactive multipath routing for large-scale MANETs (GMP-OLSR). Indian Journal of Science and Technology. 2020; 13(08), 893-906. D0l: 10.17485/ijst/2020/ v013i08/149813}

Received date: January 1,2020

Accepted date: February 10, 2020

*Author for correspondence: Kebebew Ababu Yitayih $\nabla$ kebebew. ababu@ju.edu.et $P$ Department of Electrical and Electronic Engineering, Federal University of Technology, Owerri, Imo State, Nigeria

\begin{abstract}
Objectives: To propose a group-based QoS aware proactive multipath routing based on OLSR protocol. Methodology: This work proposes a group-based proactive multipath routing based on the OLSR protocol, which is designed to be adaptive for a big crowded Mobile Ad-hoc Network to satisfy the desire for real-time multimedia applications. This approach will restrict mobile nodes to be participated in the cluster head selection process based on the mobility and energy of a node. It uses a grouping strategy to manage the network topology and performing route maintenance, and preserve multiple prioritized routes with the support of Quality of Service (QoS) aware computation. Findings: The study evaluated the performance of the proposed group-based QoS aware multipath Optimal Link State Routing (GMP-OLSR) routing through the OMNET++ network simulation environment. Within different network density, the proposed approach offered a significant reduction in end to end delay, overhead and an increment in data packet delivery ratio. Applications: Using an appropriate groupbased QoS aware multipath routing strategy for mobile ad hoc networks provides better performance and improves the services of mobile applications.
\end{abstract}

Keywords: MANET, GMP-OLSR, Clustering, Multipath Routing, QoS Aware Routing.

\section{Introduction}

Over the past few decked, computing system has evolved from large, expensive, and standalone computers to small, chip, and ubiquitous devices. These devices, different from their ancestors, they are highly mobile and have the ability to communicate with each other without requiring access points, routers, or additional networking infrastructure. This communication is formed based on Mobile ad hoc networks (MANETs) [1-5]. It is self-organizing and maintains a routing structure among them over a dynamic 
wireless interface. Due, in part, to relative movements of MANET nodes and, in part, to environmental effects, the network topology and communication links in a network may change state more frequently than in fixed wired or fixed wireless networks. In this work to find a path between the communication endpoints, the author has used the OLSR protocol. The protocol has lower latency for real-time applications, and it would be an appropriate protocol for a large-scale mobile network. However, due to high mobile node mobility, multi-hop connections, unpredictable link properties, resource constraints, disputation for link contact, and a lack of management of the network, the protocol has problems like overhead, high packet loss, and others. Therefore, in this work, the author has proposed a group-based QoS aware multipath routing approach (GMP-OLSR). This approach will restrict mobile nodes to be participated in the cluster head selection process based on the mobility and energy of a node. In order to elect as a cluster head, nodes have to belong to a super node group. This can manage the stability of the topology, maintain the route information of the network with minimum overhead, create multiple alternative routes from source to destination for a backup route, provide network traffic balancing and route link disconnection protection by spreading the data among multiple optimal paths. Since this work assures to provide efficient QoS aware communication between MANET nodes.

\section{Related Work}

Nowadays, due to the wide varieties of QoS required application in MANETs, different routing protocols have been proposed for satisfying the required services. With all research works, the challenging task is considering multiple QoS parameters that will balance the performance of the network and managing the communication of MANET nodes within limited resource consumption. Thus, this study is focused on improving the utilization of OLSR routing protocols through a group-based QoS aware multi-path route computation model. This will be applicable to multimedia applications that require QoS satisfaction.

\subsection{Attempts on Multipath Routing}

Multipath routing scheme computes multiple paths in a network that will have a big impact on the performance of the network. Many authors are introducing that using a multipath routing algorithm provides better flexibility and extensibility by employing QoS metrics. So here, some of the works which are proposed based on the OLSR protocol is assessed.

In Ref. [6], the authors proposed multipath OLSR based on the Dijkstra scheme which provides the shortest links of paths by considering cost functions. IT shows that how the services of multimedia applications are enhanced using MP-OLSR with the support of QoS. However, appropriate QoS metrics for route computation were not identified. In Ref. [7], the authors proposed multipath QOLSR based on the shortest-widest path algorithm. It enhances OLSR routing protocol for supporting multiple QoS parameters for routing. But, the author did not prove that the correlation factors of the metrics are correctly calculated. In Ref. [8], the authors proposed source routing OLSR based on IP source routing. Here, the node calculated multiple routing node-disjoint links to the destination. 
In Ref. [9], the authors also propose multipath OLSR routing to compute node-disjoint links without considering used nodes from the topology information. But, source routing has problems when we have unstable network topology. In Ref. [10], the authors propose multiple parameters such as residual energy, mobility, and link quality for multipath optimal routing in MANETs. It enhances the lifetime of the network over a sustained period of time. Considering multiple parameters is good, but here, to manage the topology of the network, the authors did not consider any strategy.

\subsection{Existing Schemes for Cluster Head Selection}

To control the node communication within a network, the MANET nodes are grouped into small clusters. Especially, when the network size and number of nodes exceeds its optimal value reduces network performance, the life of the network is short, and there is high overhead, delay, and energy consumption in the network. Thus, considering an appropriate clustering algorithm is the critical thing that has to be done. The clusters will elect cluster heads based on different criteria's such as weight of the node, node density, node ID, node degree, etc. The node which is selected as a cluster head has the role of managing within its subgroup.

There are a lot of cluster formation algorithms in the literature: HD [11], WCA [12-13] and DWCA, MANET clustering algorithms survey [14]. In Ref. [15], the author proposed a clustering-based QoS-OLSR model and relay node selection based on energy and bandwidth metrics. This has an impact on reducing relaying nodes and can efficiently prolong the network lifetime. However, the cluster formations were not applicable for topology management as it is a proactive protocol, and the clustering is not involved in the route maintenance. In Ref. [16], the paper proposed a cluster-based routing protocol for VANETs using OLSR. In this work cluster-based, QoS was introduced to reduce MPR's percentage in dense VANETs area. However, the clustering process is only focused on MPRs selection and which has a big overhead on the network during cluster formation.

\section{Materials and Methods}

As shown in Figure 1, the proposed protocol architecture is applied in MANET to enhance the services provided by the network. Each ad hoc host in the proposed protocol contains several components that will be used during the exchange of packets between the source and destination host. When MANET nodes are initiating applications that are needed to send to the destination mobile host, first it should pass through all modules of the proposed architecture. Based on an enhanced adaptive clustering algorithm, initially, each node performs clustering operation and it becomes aware of the network topology, and then to make the topology more stable, nodes which have better residual energy and similar mobility will be grouped and form supernode members for future cluster head selection. Proposing the clustering and grouping approach in MANET for a large-scale network improves the routing discovery and computation of a node within the network by reducing the size of the routing information, overhead, delay, and so on. Once the clustering 


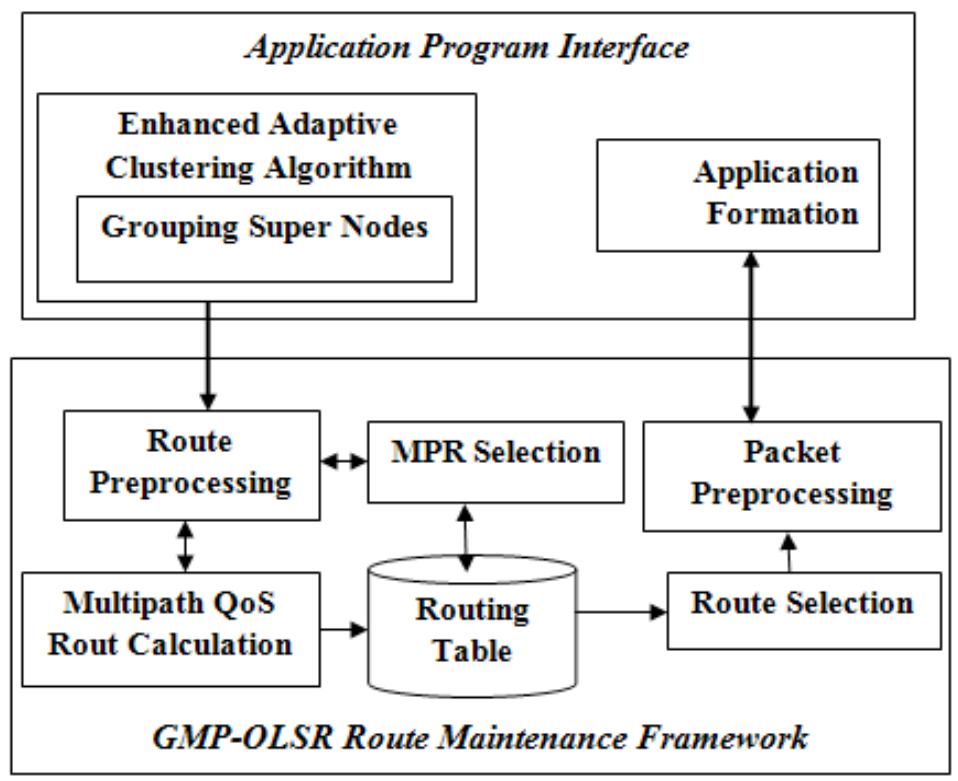

FIGURE 1. Proposed architecture.

and grouping of nodes are done, routing information of every node is maintained using QoS aware multipath Dijkstra algorithm. The algorithm considers delay, bandwidth, and link status parameters to compute the optimal path and reserved multiple paths for every destination of each node.

\subsection{Clustering}

Cluster-based routing is a good strategy to limit the routing traffic that communicates inside the network by grouping the mobile hosts into a group of clusters. Especially, when we have a big network, we should consider clustering to minimize the amount of routing control overhead and maximizing the network lifetime. In the proposed work, a groupbased clustering algorithm for grouping of MANET nodes is designed. Nodes are divided into clusters and the clustering algorithm is performed when a node joins the network. This will make the communication more efficient, scalable, and robust than the existing OLSR routing protocols. The cluster is performed based on QoS metrics of nodes within the transmission range, and each node can be in one of the three states which are cluster head, ordinary, or gateway [17]. The main objective of creating clusters on a MANET is, by limiting the network view of each node, we can minimize the routing overhead of broadcasting messages. Moreover, the movement of nodes is limited only within the intracluster zone without affecting other parts of the network and so the performance of the network is highly increased. However, when the topology is changed, there should be a re-clustering and every node again will be part of the clustering process. That will have a big challenge in the case of a large-scale network. Therefore, in our proposed work, based on mobility and residual energy, this study proposed a technique to restrict nodes participating in the cluster head selection. The grouping strategy will select supernodes 
which are capable to be selected as cluster head. This will limit the number of control packets exchanging between nodes, the computational resource, and channel utilization wasted during cluster maintenance.

To maintain the network topology, this study proposes an enhanced adaptive clustering algorithm. The proposed approach is working based on QoS support parameters for providing an efficient route for an application that requires QoS support like multimedia data. The algorithm will adapt Lin's cluster formulation, which is the low cost of the cluster maintenance algorithm enhanced adaptive clustering algorithm rather than considering the lowest ID as the only parameter for cluster formation, it will consider the neighbor node connectivity of a node having a direct link. Considering node density of a node will solve frequent re-clustering and change of cluster heads, and make the topology more stable. Especially when we have a big network, the approach should consider the stability of the network. The node connectivity information is generated based on Hello Messages during the pre-phase of cluster formation.

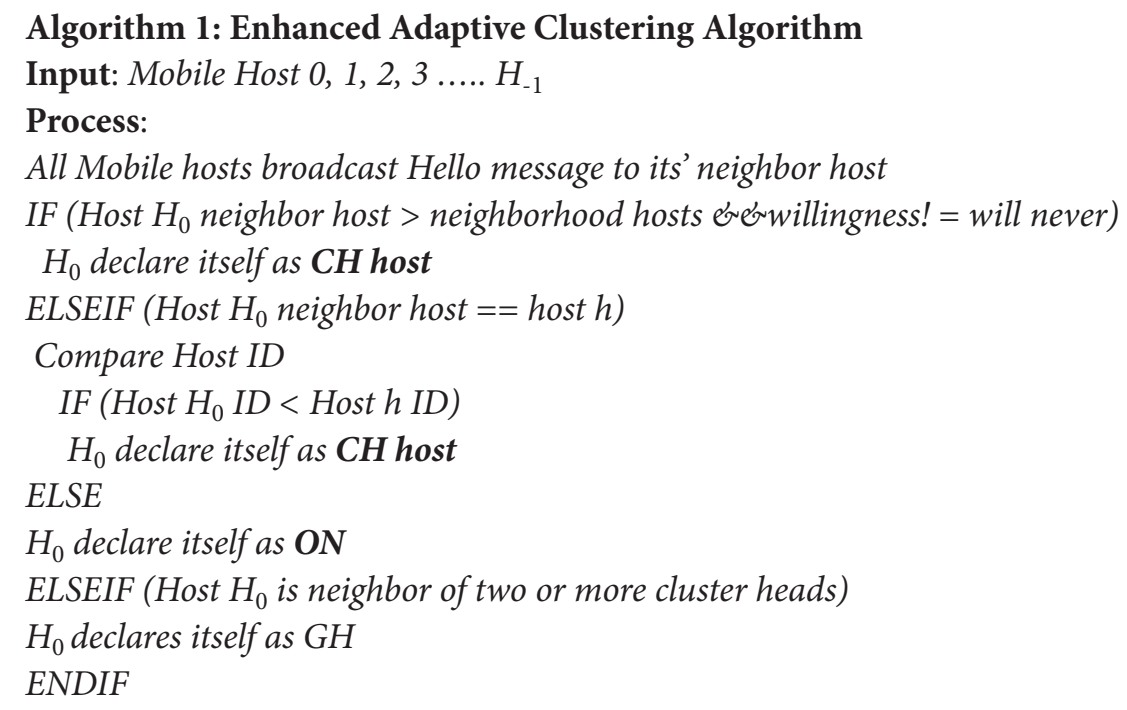

Output: Cluster head hosts (CH), Gateway host (GH), and Ordinary nodes (ON)

\subsection{Grouping Super Nodes}

Supernode groups, hold nodes that are only participating for future selection of cluster head without wasting computational power, control packet, and other resources. All cluster nodes communicate with a cluster head using grouping messages to find out the energy and mobility of a node based on the following equations.

After a simulation is running, the energy consumption of by a particular node at time $t$ $(E(t))$ is computed as follows,

$$
E(t)=D P_{\mathrm{tx}}^{*} \alpha+D P_{\mathrm{rx}}^{*} \beta
$$

${ }^{\star} D P_{\mathrm{tx}}=\#$ data packets transmitted by the node after time $t$

${ }^{\star} D P_{\mathrm{rx}}=\#$ data packets received by the node after time $t$ 
${ }^{*} \alpha$ and $\beta$ are constant values in the range of $[0,1]$

If $E_{\mathrm{i}}$ denotes the initial energy of a node, the residual energy RE of a node at time $t$, can be calculated as:

$$
\mathrm{RE}=E_{\mathrm{i}}-E(t)
$$

Then the average residual energy of a node is calculated as:

$$
\mathrm{RE}_{\mathrm{avg}}=\sum_{n=1}^{n}\left(\frac{R E_{i}+\ldots R E_{n}}{n}\right)
$$

The second parameter that the author considering for selecting supernodes is mobility. Mobility is the combined functions of movement patterns of a node, relative mobility of node, and movement direction. A node has the same relative mobility, speed, and direction will have a high probability to become a member of supernodes.

If the $\mathrm{RE}$ of host $i$ is greater than the $\mathrm{RE}_{\mathrm{avg}}$ and the mobility of the node is similar, then host $i$ become the member of a supergroup. Otherwise, host $i$ cannot be part of a supernode group. Furthermore, if the node residual energy is less than a certain threshold value, the member node will be erased from the group.

To conserve the resource utilization of node for selection of cluster head in the future, the cluster head will be automatically selected from these supergroup set of nodes based on residual energy and mobility comparison rather involving all other nodes.

\subsection{QoS Metrics for MP_OLSR}

To compute the routing paths for each destination, the author has considered appropriate multiple QoS metrics that would satisfy the real-time communication. These metrics are delay, bandwidth, and quality of the link.

a) Link Delay: It shows how long it takes for a bit of data to travel across the mobile adhoc network from one source host to the endpoint of another host. It is the total time that taken between two nodes for which a source node $\mathrm{n} 1$ sends a message to destination node $\mathrm{n} 2$ and successfully delivered. This metric is important in delay-sensitive applications such as video and voice transmission. In Eq. (1), the author has shown the formula for calculating a delay of nodes.

$$
\text { Delay }=\sum_{0}^{n}\left(\frac{\text { Recievedtime }- \text { Senttime }}{\mathrm{n}}\right)
$$

${ }^{*} n$ is number of packets received

b) Link Quality: It shows the capability of the link between mobile hosts. The quality of a link is computed based on a link quality indicator. It indicates the quality of the packets received during periodical exchanges of the Hello messages between nodes and its neighbors in a certain time interval. The received signal strength (RSS) is used to 
measure the signal quality. The links must be checked in both directions in order to be considered valid. The path that has the bigger link quality indicator has a better chance of delivering a message to the destination.

c) Link state: it is the technique in which each host shares information with another host about the reach-ability of other networks and the metric to reach the other networks in order to determine the best path. It is working based on the status of the link at time $t$, when $t$ is referred to as the estimation period (namely, the time required for estimating available bandwidth). Eq (5) shows the formula for calculating the link status.

$$
S i(t)=\frac{(T A B-T D S)}{T A B}
$$

$S_{\mathrm{i}}(t)=0$ or less than 0 , link is busy,

Greater than 0, link is idle

TAB is the total available bandwidth, and TDS is the transmitted data size at a time $t$.

To compute the available link bandwidth of hosts [17] uses idle time. As shown in Eq. (5), the link-state is calculated by subtracting the transmitted data size from the total available bandwidth and dividing it by the available bandwidth. Therefore, based on the above values, QoS metrics is computed as in Eq. (6):

$$
\text { QoS Metrics }=(\mathrm{LQ}+\mathrm{ALBW})-\mathrm{LD}
$$

${ }^{*} L Q=$ Link Quality, ALBW=Available link bandwidth, LD = Link Delay

Algorithm 2 shows the proposed QoS aware route computation algorithm based on the existing multipath Dijkstra scheme. The proposed algorithm is working by considering multiple QoS metrics that will satisfy the services of real-time applications. The node executes this algorithm to obtain the multiple paths to the destination of all other nodes, and store it in the multipath routing table.

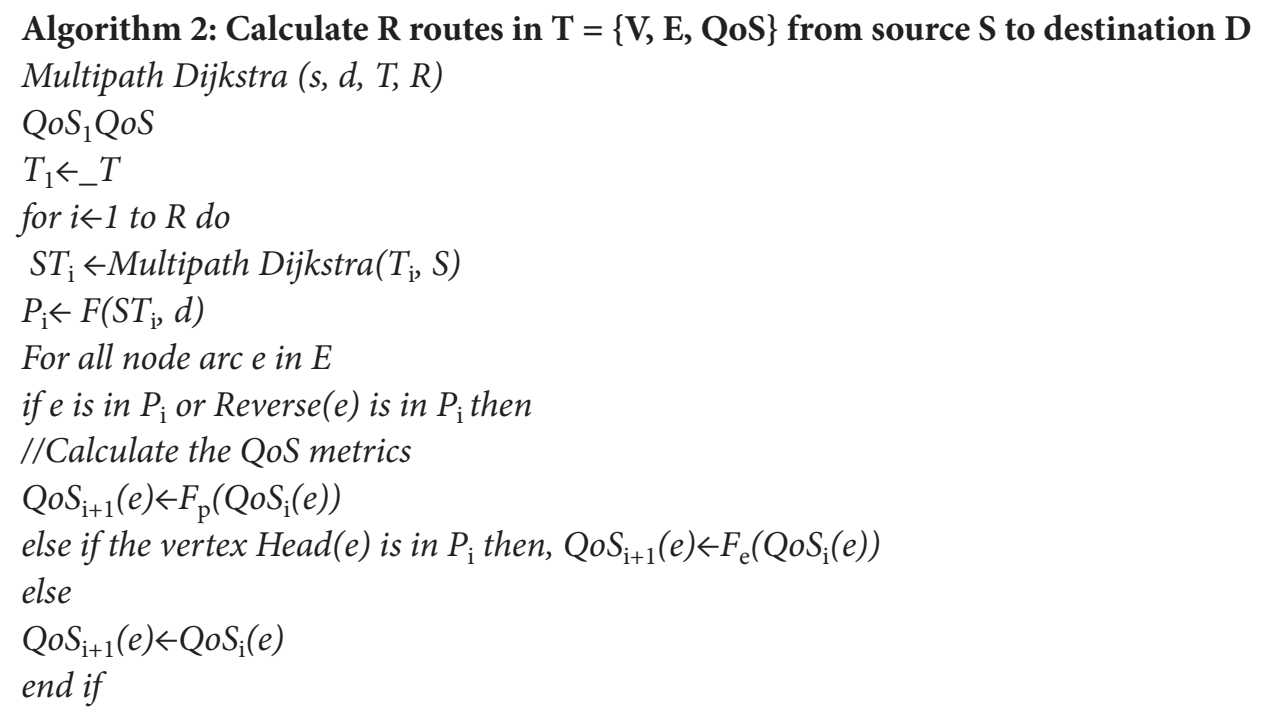




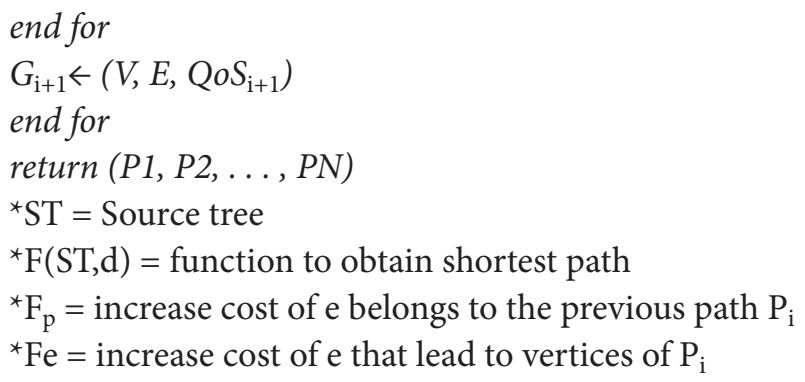

\subsection{Cluster-based QoS MP OLSR Route Maintenance}

In order to communicate and transmit a packet in a mobile ad-hoc network, this work proposes QoS aware multipath Dijkstra algorithm. First, each host calculates an optimal routing path to the destination of every other node using as shown in algorithm 2 . Once the optimal path is computed, it will be stored in the routing table of the source node based on Algorithm 3.

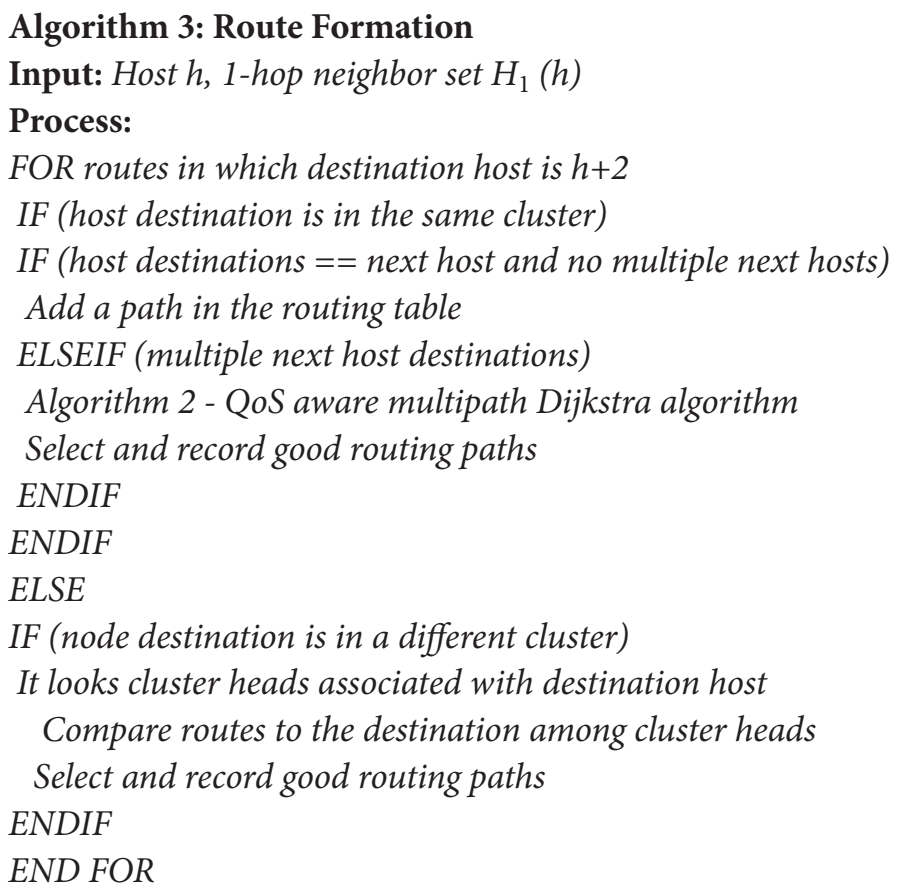

Output: Routing Table

\section{Network Simulation and Experimental Results}

To evaluate the performance of the proposed adaptive group based QoS aware multipath routing protocol in a large-scale mobile ad-hoc network, the author has been implemented in a simulation using the latest version of OMNET++ with INET framework [18]. 
Data collection was done as the simulation is running using OMNET++ data collection APIs. OMNET++ also provides analysis tools to analyze the collected data. Figures 2 and 3 show the simulation of mobile ad-hoc hosts using the proposed approach.

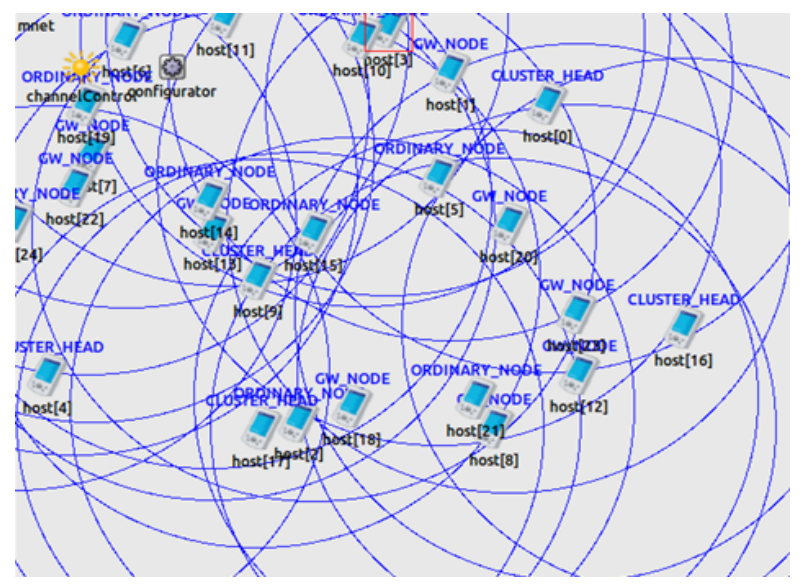

FIGURE 2. Proposed MANET simulation.

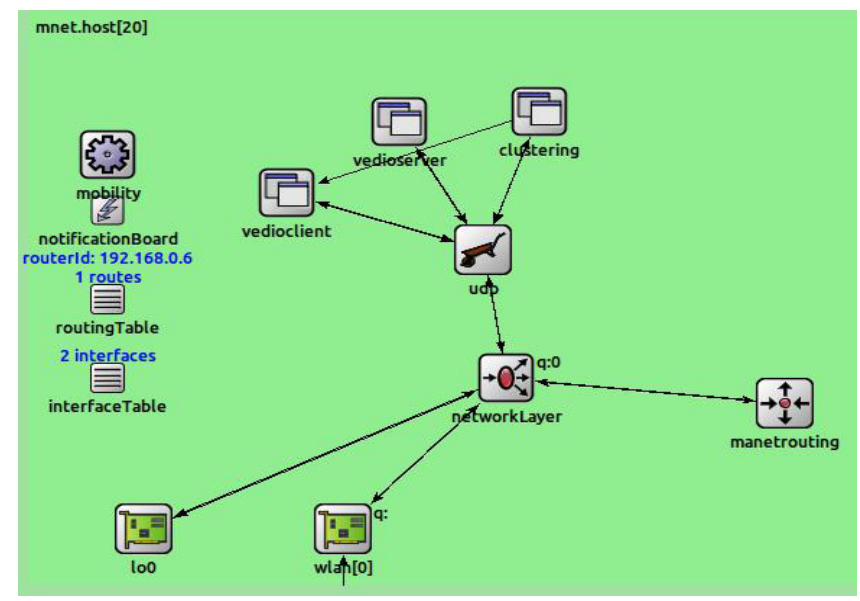

FIGURE 3. Adhoc host setup.

The algorithm will be tested with different node sizes, each of which will participate in cluster formation and then sending of test packets to nodes destined within the same cluster and outside of the cluster. The clustering algorithm is implemented using UDP sockets to minimize resource footprint. Packet delivery assurance is handled by the clustering algorithm using timeouts.

\section{Result and Discussion}

In order to route a packet with QoS support, the author has proposed two schemes and evaluated them with the existing approach. Our proposed methods are 1) Cluster-based Multipath Optimal Link State Routing (CMP-OLSR) which is working based on my 
TABLE 1. Simulation parameters

\begin{tabular}{ll}
\hline Experimental parameter & Value \\
\hline \#MANET nodes & $70,100,130,150$ \\
Area & $600 \mathrm{~m} \times 400 \mathrm{~m}$ \\
Total simulation time & 3600 seconds \\
Wireless link speed (bit rate) & $2 \mathrm{Mbps}$ \\
Wi-Fi standard & IEEE $802.11 \mathrm{n}$ \\
Mobility & Mass mobility model \\
Data traffic & CBR, UDP \\
\hline
\end{tabular}

proposed adaptive clustering approach, 2) Group-based Multipath Optimal Link State Routing (GMP-OLSR) schemes, and here in addition to clustering the author has applied a group approach for supper node selection that will participate during cluster head selection. These two approaches are evaluated with the existing MP-OLSR strategies based on Average End to End Delay (AE2ED), Packet Delivery Ratio (PDR), Throughput, and Normalized Routing Load (NRL).

The vector and scalar data of the simulation are collected through the OMNET++ API and analyzed as shown in Table 2. As we can observe from the table, within different network sizes, considering the topology management strategy provides better performances in all evaluation criteria than the existing strategies. The collected spreadsheet data are demonstrated in a graph as shown in Figures 4-7.

Figure 4 shows the performance of the average E2E delay between three algorithms by increasing the number of mobile hosts from 70 to 150 . This result has been gotten after the simulation time of 3600 seconds with each network size. From the graph, we can observe that in all network sizes, GMP-OLSR and CMP-OLSR strategies result in lower Average E2E delay than existing non-cluster MP-OLSR. This is because of the clustering scheme, the network is more manageable, creates fewer intermediate nodes, easy to maintain the optimal route and each node maintains stable routing information and has fewer disconnection paths. Still, when we increase the network size the topology updates

TABLE 2. Performance evaluation result

\begin{tabular}{lccccccc}
\hline \multirow{2}{*}{$\begin{array}{c}\text { Selection } \\
\text { approach }\end{array}$} & \multirow{2}{*}{$\begin{array}{c}\text { Network } \\
\text { size }\end{array}$} & \multirow{2}{*}{$\begin{array}{c}\text { Total } \\
\text { (\#node) }\end{array}$} & $\begin{array}{c}\text { Total packet } \\
\text { (\#) }\end{array}$ & \multicolumn{4}{c}{ Performance } \\
\cline { 6 - 8 } & (\#packet) & delivered & (\#packet) & PDR & AEED & & THP \\
& 70 & 479 & 340 & 71 & 32.18 & 2.56 & 419.35 \\
& 100 & 539 & 371 & 69 & 37.09 & 2.76 & 578.98 \\
& 130 & 793 & 459 & 58 & 41.09 & 2.81 & 592.1 \\
MP-OLSR & 150 & 801 & 451 & 56 & 45.32 & 2.98 & 612.68 \\
& 70 & 460 & 385 & 84 & 25.79 & 1.96 & 517.67 \\
& 100 & 645 & 489 & 76 & 28.37 & 2.15 & 629.76 \\
& 130 & 806 & 579 & 72 & 32.5 & 2.35 & 638.47 \\
CMP-OLSR & 150 & 811 & 571 & 70 & 34.25 & 2.41 & 648.56 \\
& 70 & 481 & 411 & 85 & 22.79 & 1.56 & 531.62 \\
GMP-OLSR & 100 & 605 & 486 & 80 & 26.17 & 1.96 & 647.71 \\
& 130 & 826 & 639 & 77 & 28.79 & 2.16 & 657.6 \\
& 150 & 796 & 585 & 73 & 31.01 & 2.24 & 667.82 \\
\hline
\end{tabular}




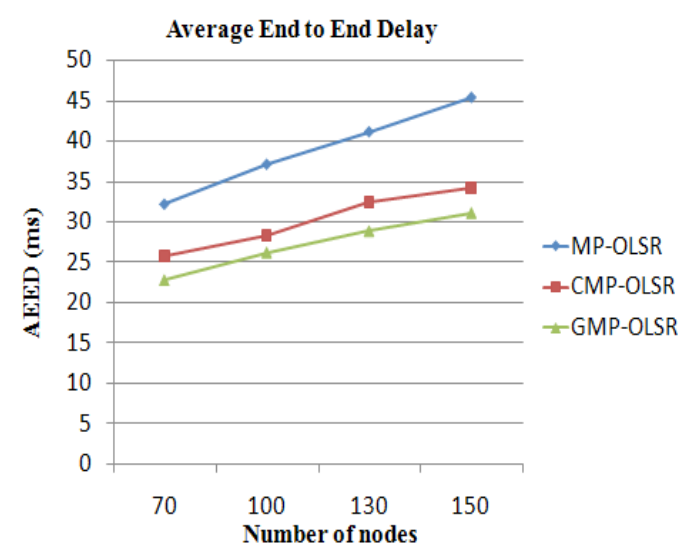

FIGURE 4. Average E2E delay.

to maintain the cluster information make the network more crowded and create high congestion and delay of a packet. So, the group-based approach during cluster formation limits the exchange of information for cluster formation to specific nodes without affecting the whole network. Due to that GMP-OLSR shows less average E2E delay than others. Additionally, in the proposed GMP-OLSR and CMP-OLSR schemes, QoS aware multipath computation was conducted and delay is among the constraints that considered for selecting optimal disjoint-paths. This result the proposed algorithm improves the delivery of data traffic more than MP-OLSR.

Figure 5 shows that the PDR for the GMP-OLSR and CMP-OLSR are better that MP-OLSR, especially when we have less number of network size, both algorithms have a better packet delivery. However, when the network size increases, there will be a high probability of path breakage and congestion of packets that result in high packet loss. The packet delivery ratio for QoS routing schemes decreases as the size of the network increases. In GMP-OLSR, the PDR dropped from $85 \%$ to $73 \%$ while others also dropped highly. However, when we see the ratio of packet loss among all algorithms with varied network sizes, in GMP-OLSR, the dropping result is very minimal.

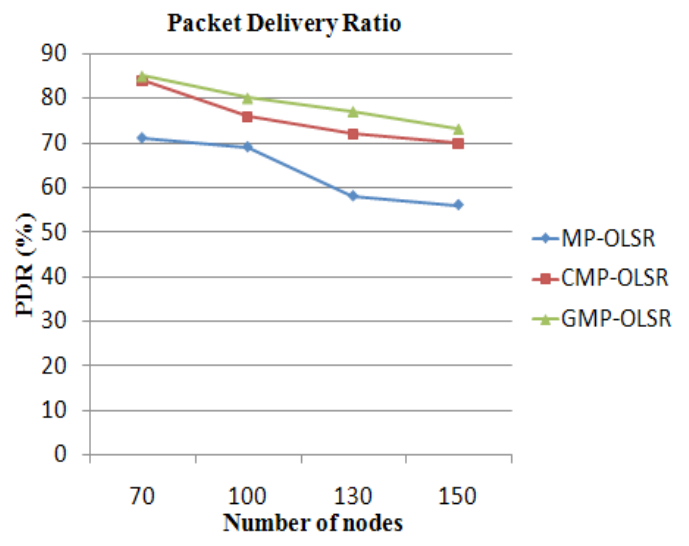

FIGURE 5. Packet delivery ratio. 
Figure 6 shows the routing control packets among the three algorithms sent by all MANET nodes over the number of received data packets at the destination. MP-OLSR has a high flooding rate of multicasting and broadcasting messages compare with the proposed models. CMP-OLSR and GMP-OLSR minimize the overhead of a network by limiting the number of control packets retransmitted to a specific group. GMP-OLSR limits participants' nodes for cluster formation which yields lower control packet exchanging between nodes. Therefore, GMP-OLSR results in lower routing overhead than the others.

Figure 7 shows the difference in data delivery over a given time period among the three algorithms. In GMP-OLSR, the network topology is well managed and the result shows that the number of data packets that are delivered in a pre-defined time is better than the others. In all simulations, the experiment result shows that the proposed group-based QoS aware routing approach provides better performance for applications that require QoS support such as Voice over IP, Video conferencing, live streaming, etc., with high packet delivery ratio and throughput, and minimum routing load and E2E delay.

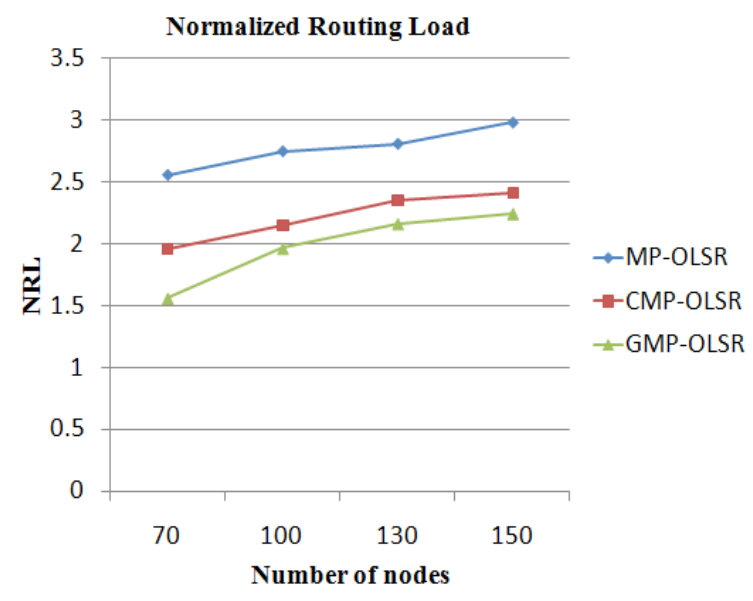

FIGURE 6. Normalized routing load.

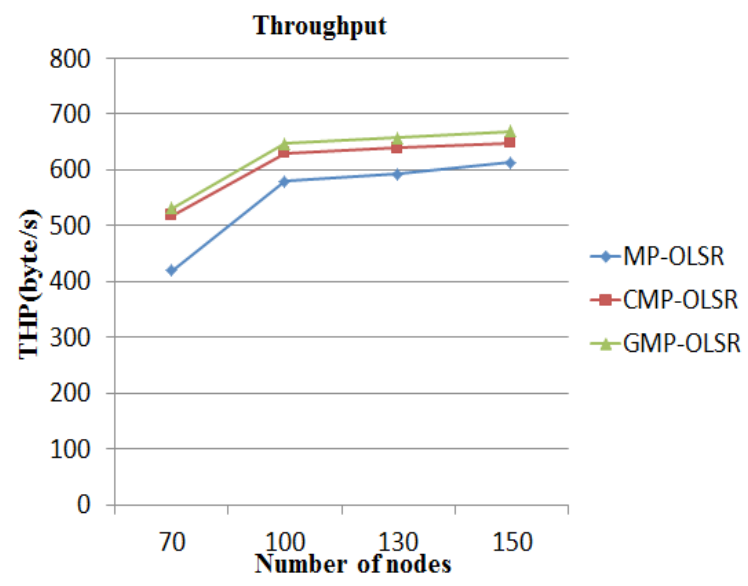

FIGURE 7. Throughput. 


\section{Conclusion}

In MANET, QoS aware multimedia communication for real-time communication is very challenging. In this study, we are proposing a group-based cluster formation approach for managing the topology of the network. The cluster is performed based on node connectivity and lowest ID metrics. And the grouping strategy is applied to select supper mobile nodes based on mobility and residual energy. This will restrict nodes participating in the cluster head selection and minimize the amount of control packet. Proposing the clustering and grouping approach in MANET for a large-scale network improves the routing discovery and computation of a node within the network by reducing the size of the routing information, overhead, delay and so on. Once the clustering and grouping of nodes are done, routing information of every node is maintained using QoS aware multipath Dijkstra algorithm. The algorithm considers delay, bandwidth and link status parameters to compute the optimal path and reserved multiple paths for every destination of each node. Generally, in this study, we have proposed QoS aware multipath routing, adaptive clustering algorithm, and group-based supernode selection strategies for improving the services of MANET application that requires QoS support. Applying these schemes in a large-scale network enhances the performance of the existing OLSR routing protocol by minimizing problems and reducing the multicast and broadcast packets. The routing approaches have been evaluated with each other. On average, GMP-OLSR has $78.75 \%$ PDR and $27.19 \mathrm{~ms}$ average E2ED while MP-OLSR is 63.5\% PDR and $38.92 \mathrm{~ms}$ average E2ED. Thus, GMP-OLSR performs better result in all evaluation parameters and it will be appropriate for real time-sensitive multimedia applications which require QoS support such as Voice over IP, Video conferencing, live streaming, etc, with high packet delivery ratio and throughput, and minimum routing load and E2E delay. Additionally, the author suggests that considering the security aspects of the protocol would enhance the services in MANETs.

\section{Data Availability}

The research file (source code) of the author's work is publically available at Github. https:// github.com/kebebewss2/QoS-routing-in-MANET-using-OMNET-

\section{Acknowledgments}

The research work is done at Jimma University, Ethiopia. The author thanks the Jimma University, Research Director Office and Faculty of computing for supporting through different resources.

\section{References}

1. Rath M, Pati B, Pattanayak BK. Energy efficient MANET Protocol using cross-layer design for military applications. Defense Science Journal. 2016, 66(2). DOI: 10.14429/dsj.66.9705. 
2. Tonnesen A. Implementing and extending the optimized link-state routing protocol, Master's thesis, University of Oslo, Norway. 2004; 1-55. http://www.olsr.org/docs/master-pres.pdf

3. Gong S. Quality of service aware routing protocols for mobile ad hoc networks. IEEE Journal. 2006, 698(27). http://lib.tkk.fi/Dipl/2006/urn007361.pdf

4. Ge Y, Kunz T, Lamont L. Quality of service routing in Ad hoc networks using OLSR. In: System sciences. Proceedings of the 36th annual Hawaii international conference. 2003, 9. https:// www.researchgate.net/publication/221182522_Quality_of_Service_Routing_in_Ad_Hoc_ Networks_Using_OLSR

5. Badis H, Munaretto A, Al Agha K, Pujolle G. Optimal path selection in a link-state QoS routing protocol. Proceedings of the IEEE 59th vehicular technology conference. 2004, 5, 2570-2574. https://doi.org/10.1109/VETECS.2004.1390660

6. Badis H, Agha KA. Qolsr multi-path routing for mobile ad hoc networks based on multiple metrics: bandwidth and delay. In: IEEE Vehicular Technology Conference. 2004; 2181-2184. https://doi.org/10.1109/VETECS.2004.1390660

7. Kun M, Jingdong Y, Zhi R. The research and simulation of multipath OLSR for mobile ad hoc network. In: International symposium on communications and information technologies. 2005; 540-543. DOI: 10.1109/ISCIT.2005.1566912.

8. Zhou X, Lu Y, Xi B. A novel routing protocol for ad hoc sensor networks using multiple disjoint paths. In: 2nd International conference on broadband networks. Boston, MA, USA. 2005. https://doi.org/10.1109/ICBN.2005.1589706

9. Ali C, Ahmed H, El Koutbi M. Efficient heuristic based on clustering approach for OLSR. Journal of Computer Networks and Communications. 2013, 7. https://doi.org/10.1155/2013/597461

10. Tilwari V, Dimyati K, MHD Nour Hindia, Anas Fattouh, Sadegh Amiri I. Mobility, residual energy, and link quality aware multipath routing in MANETs with Q-learning algorithm. MDPI, Applied Science. 2019, 9(8), 1582. DOI: 10.3390/app9081582.

11. Bentaleb A, Boubetra A, Haros S. Survey of clustering schemes in mobile ad hoc networks. Communications and Network. 2013, 5(2). https://www.comp.nus.edu.sg/ bentaleb/files/ papers/journal/AdhocSurvey.pdf

12. Baccelli and Emmanuel. OLSR trees: a simple clustering mechanism for OLSR. In: Challenges in ad hoc networking. 2006; 265-274. https://hal.inria.fr/hal-00775902/document

13. Bakht A, Humayun D. Survey of routing protocols for a mobile ad-hoc network. International Journal of Information and Communication Technology Research. 2011, 1-13. https://pdfs. semanticscholar.org/7b7c/6825fc269a0d0d15d6a82fffaaad1806151d.pdf

14. Yu JY, Chong PHJ. A survey of clustering schemes for mobile ad hoc networks. IEEE Communications Surveys and Tutorials. 2005, 7(1-4), 32-48. https://doi.org/10.1109/COMST.2005.1423333

15. Hadi Otrok, Azzam Mourad, Jean-Marc Robert, Nadia Moati, Hiba Sanadiki. A clusterbased model for QoS-OLSR protocol. In: 7th, IWCMC. 2011. https://doi.org/10.1109/ IWCMC.2011.5982694

16. Kadadha M, Hadi Otrok, Hassan Barad, Al-Qutayri M, Yousof Al-Hammadi. A cluster-based QoS-OLSR protocol for urban vehicular ad hoc networks. In: IWCMC. 2018. https://doi. org/10.1109/IWCMC.2018.8450405

17. Yitayih KA, Libsie M. Towards developing enhanced cluster-based QoS-aware routing in MANET, Hindawi. Journal of Computer Networks and Communications. 2020, 10. https://doi. org/10.1155/2020/5481916

18. Canourgues L, Lephay J, Soyer L, Andre-Luc Beylot. Scalable adaptation of the OLSR protocol for large clustered mobile ad hoc networks. IFIP International Federation for Information Processing, Advances in Ad Hoc Networking. 2008, 265, 97-108. https://link.springer.com/ chapter/10.1007/978-0-387-09490-8_9 